\title{
Auditoría Integral En La Contratación De Servidores Públicos En El Gobierno Regional De Amazonas Periodo 2020
}

Comprehensive Audit In The Hiring Of Public Servants In The Regional Government Of Amazonas Period 2020

\section{Juana del Pilar Contreras Portocarrero}

Maestro en Gestión Pública, Universidad Cesar Vallejo, Lima, Perú

Pilar_contreras@msn.com, ORCID 000-0001-7201-8996

https://scholar.google.es/citations?user=QKvd2T8AAAAJ\&hl=es

\section{Yohnny Huárac Quispe}

Maestro en Gestión Pública, Universidad Cesar Vallejo, Lima, Perú

yhuaracq@hotmail.com, ORCID 0000-0002-5558-1979

https://scholar.google.com/citations?view_op=list_works\&hl=es\&user=Xw

FFk-IAAAAJ

\section{Fausto Gilberto Blas Ghiggo}

Maestro en Gestión Pública, Universidad Cesar Vallejo, Lima Perú

Faustoblas2406@gmail.com,ORCID 0000-0001-8414-9416

https://scholar.google.es/citations?user=41KSW5UAAAAJ\&hl=es

\section{Sherley Catherine Morillo Pereda}

Maestra en Gestión Pública y Gobernabilidad, Universidad Cesar Vallejo,

Lima, Perú

morillo_s@hotmail.com, ORCID: 0000-0002-1332-7497

https://scholar.google.es/citations?user=eq0pBmYAAAAJ\&hl=es 
Resumen: El presente artículo tiene por objetivo hacer una correlación sobre la auditoría integral y la contratación de servidores público en el Gobierno Regional de Amazonas. Inicialmente se plantean algunos antecedentes relacionados con la auditoría integral y la contratación de servidores públicos; posteriormente se realiza una conceptualización de la Auditoría integral como herramienta para mitigar riesgos y tomar decisiones oportunas para el logro de los objetivos de la institución, Se concluyó que existe evidencia para afirmar que la auditoría integral se relaciona significativamente con la contratación de servidores públicos, siendo que el coeficiente de correlación Rho de Spearman de 0.9.10, en el cual se presenta una correlación alta entre las variables. Palabras Clave: auditoria integral, contratación de servidores públicos

Abstrac: The objective of this article is to make a correlation on the integral audit and the hiring of public servants in the Regional Government of Amazonas. Initially, some antecedents are raised related to the comprehensive audit and the hiring of public servants; Subsequently, a conceptualization of the Comprehensive Audit is carried out as a tool to mitigate risks and make timely decisions to achieve the institution's objectives.It was concluded that there is evidence to affirm that the Comprehensive Audit is significantly related to the hiring of public servants, being than Spearman's Rho correlation coefficient of $0.9,10$, in which there is a high correlation between the variables. Keywords: comprehensive audit, hiring of public servants 
Introducción:En la presente investigación se pretende establecer si la auditoría integral y la contratación de servidores públicos en el gobierno regional de Amazonas están relacionadas.

En el ámbito internacional y, La Auditoría Integral, no es la suma de las auditorías, sino va integrar a las auditorías, es decir es una auditoria multifuncionaria, pero el contador es el único profesional idóneo quién dará una seguridad razonable a los estados financieros, teniendo en cuenta el marco conceptual de información financiera. Para dar esta opinión o dictamen se enmarcarán en las normas de auditoría generalmente aceptadas NIC y NIFF. (Blanco, 2015)

La implementación de una Auditoría Integral contribuye a mitigar riesgos y tomar decisiones oportunas, porque los cambios son constantes en la economía mundial en consecuencia generan nuevos requerimientos de la información que emite una organización, más aún si su actividad depende de negociaciones internacionales en las cuales clientes o proveedores residen en el exterior, entonces, la información que se intercambia con estos debe estar presentada bajo estándares internacionales que permitan un análisis transversal de la misma.(Ramírez, 2017).

La aplicación de la Auditoría Integral las organizaciones del Perú, contribuirá a evaluar objetivamente, en un periodo determinado, evidencias relativas a la información financiera al comportamiento económico y al manejo de organizaciones, con el propósito de informar sobre el grado de correspondencia entre aquellos y los criterios de los indicadores establecidos o los comportamientos generalizados. (Falcon, Quipukamayoc).

Por otro lado, (Bautista, 2009), manifiesta que "La Auditoría Integral implica la ejecución de un trabajo con el trabajo o enfoque, por analogía de las revisiones financieras, de cumplimiento, control interno y de gestión, sistema y medio ambiente". Esta auditoría determinará si los EEFF son presentados 
de acuerdos a las NIC, si el CI de la organización contribuye al cumplimiento y/o logro de los objetivos previstos por el ente y el grado de eficiencia y eficacia con que se han manejado los recursos disponibles.

En el plano nacional, la contratación de servidores administrativos surge por la necesidad de una organización para desarrollar diferentes actividades y/o servicios, asimismo establece procedimientos, normativas, con la finalidad de cumplir los objetivos de lao organización, asimismo, mitigar errores, fraudes, pagos ilegales, malversaciones y otras prácticas delictivas prestando mayor atención al establecimiento de mejores controles internos para dar respuesta a un conjunto de inquietudes. (Fudkhoury, 2014)

Se considera como dimensión de auditoría integral: auditoría financiera, auditoría gestión, auditoría de cumplimiento y auditoría de control interno La Ley del servicio civil N³0057, hace alusión que "la contratación de los servidores público es con el único y exclusivo para las personas que prestan servicios en las entidades públicas del Estado, con el propósito que el estado alcancen mayores niveles de eficacia y eficiencia, y presten efectivamente servicios de calidad a través de un mejor Servicio Civil, así como promover el desarrollo de las personas que lo integran".

Asimismo, hace mención de los siguientes principios: Interés general, eficacia y eficiencia, igualdad de oportunidades, mérito, provisión presupuestaria, legalidad y especialidad normativa, transparencia, rendición de cuentas de la gestión, probidad y ética pública, flexibilidad, protección contra el término arbitrario del Servicio Civil. La presente Ley otorga al servido

Según, la Directiva 001-2019-SERVIR/GDSRH, Normas para la Gestión de los Procesos de Selección en el Régimen de la Ley N³0057, Ley del Servicio Civil. 
Está ley establece normas técnicas, métodos y procedimientos de cumplimiento obligatorio en materia de gestión de procesos de selección del régimen de la Ley $\mathrm{N}^{\mathrm{o}}$ 30057, Ley en mención expone que la convocatoria de contratación administrativa de servicios nace de acuerdo a las necesidades del puesto o cargo, por lo tanto, cuenta con requisitos para su celebración del contrato de servidores públicos.

$\checkmark$ Requerimiento realizado por la dependencia usuaria.

$\checkmark$ Disponibilidad presupuestaria, determinada por la oficina de presupuesto de la entidad o quien haga sus veces. Asimismo, los requerimientos se darán de la siguiente manera: a. Perfil del puesto. Corresponderá a cada entidad o área determinar los perfiles del puesto de acuerdo a las necesidades particulares del puesto y/o cargo materia de convocatoria, b. Características del puesto y/o cargo. Comprende la descripción de las funciones y actividades a realizar en el puesto y/o cargo, c. Condiciones esenciales del contrato. La relación laboral bajo el régimen laboral; el lugar de prestación del servicio, duración y monto de la remuneración, d. Cronograma y etapas del proceso de selección que comprende los siguientes lineamientos.

1. Etapa preparatoria. Aprobación de la convocatoria y publicar en el MINTRA (10 Días hábiles)

2. Convocatoria. Publicar en la entidad solicitante 5 días hábiles mural o web y presentación de hoja de vida.

3. Etapa de Selección. Evaluación de hoja de vida, técnica psicológica, etc. y entrevista.

En el país de Colombia, (Rincon Patiño, Rafael. 2005), hace mención que "la corrupción es uno de los mayores flagelos que azota a la sociedad colombiana y afecta principalmente a los sectores más empobrecidos, pues es a ellos a 
quienes se les soslaya los derechos fundamentales reconocidos por la Constitución Política, negándoles la posibilidad democrática de una vida digna y un desarrollo humano integral". En consiguiente se entiende como corrupción como uno de los mayores obstáculos en el cumplimiento de los fines del Estado, particularmente el servicio a la comunidad, la promoción de la prosperidad, la implementación de la participación ciudadana, el aseguramiento de la convivencia pacífica y la vigencia de un orden justo.

\section{Investigaciones internacionales}

Se han examinado y tomado en cuenta las siguientes investigaciones previas del ámbito internacionales relacionados a la auditoría integral, y de acuerdo con (Acosta, 2011), “en su investigación menciona los métodos bibliométricos y técnicas de visualización basadas en el análisis de redes sociales para analizar y procesar los datos; el análisis-síntesis para conformar la base teórica y metodológica, así como el sistémico-estructural para abordar las relaciones entre auditorías de información y auditorías de conocimiento, con un enfoque integral”. (Cedeño, 2016), desde el punto de vista metodológico “ esta investigación genera la aplicación de conocimiento válido y confiable para TSC en sus áreas administrativas financiera, mediante la adopción de un instrumento como lo auditoría integral para evaluar su desempeño". , Por lo tanto la implementación de una Auditoría Integral en TUNASERV Servicios Portuarios S.A., logrará mitigar deficiencias en las áreas contable, comercial y administrativa que afectan de forma negativa el adecuado y eficiente desempeño de la empresa, por lo tanto las capacitación y competencias profesionales son factores fundamentales para el logro de los objetivos institucional, y aplicar los resultados de este trabajo como una herramienta que contribuya en la gestión y toma de decisiones. (Veloz, et al 
2017), la presente investigación en su metodología es de "corte exploratorio, investigación descriptiva, asimismo, en sus conclusiones considera que la implementación de una Auditoría Integral demuestra validez y pertinencia en calidad de herramienta gerencial aplicada a las pequeñas Cooperativas de Ahorro y Crédito, al realizar un diagnóstico de la situación actual, se evalúa y se plantea un plan de mejoras para llegar al cumplimiento de los objetivos de la organización”. (Bautista, 2015), concluye que es importante “establecer la enseñanza ética en la formación de los servidores públicos en tres momentos fundamentales: Durante la formación académica; mediante el proceso de Inducción al servicio público y mediante un sistema basado en calidad de Capacitación y Desarrollo de Personal". Por otro lado, en acuerdo con (VACA 2017), que, el objetivo de "relacionar los factores de motivación laboral que describen el comportamiento de los servidores públicos en la última década, a través del análisis de varias teorías de motivación que permitan determinar los factores que más motivan a un servidor público del país".

\section{Investigaciones nacionales}

Con relación a los trabajos previos nacionales podemos mencionar: Guardia (2020), quien estableció la relación entre variables; su población fue conformada por 135 participantes y llegó a la siguiente conclusión: La manera como alcanzar un alto grado de suficiencia en la auditoría integral de las empresas; es mediante la mayor cantidad de papeles de trabajo, porque de esa forma se obtiene la información que deberá compararse con los criterios correspondientes. De la misma manera, Campos (2019), determinó un "método descriptivo y explicativo, siendo su población de 42 trabajadores entre, directivos, funcionarios y trabajadores del área de abastecimientos y servicios auxiliares de la Universidad Nacional Daniel Alcides Carrión”. 
Asimismo, Aspajo (2012), en el presente trabajo de investigación corresponde al "diseño descriptivo explicativo causal compuesta, no experimental, por ser eminentemente de causa - efecto entre la auditoría integral y la dirección financiera del Hospital Carlos Monge Medrano de la ciudad de Juliaca en el año 2016, su población fue de 69 trabajadores”. Príncipe (2019), Fue una investigación "descriptiva inferencial. La muestra fue de 120 personas y se identificó que hay una relación regular”.

\section{Teoría de Auditoría Integral.}

Entre las teorías relacionadas a la auditoría integral. Dice, (Bautista, 2009). Que el ejercicio de la Auditoría Integral se desarrolle en un ambiente controlado, es importante conducirla dentro de un concepto de normas que provean una estructura, como la posibilidad de pronosticar los resultados la cual ayudará a desarrollar una auditoría de alta calidad respondiendo a la necesidad de completar tareas difíciles en forma oportuna, garantizando la veracidad de los hallazgos y el soporte adecuado para las recomendaciones. Asimismo, (Luna, 2015). Expone que una auditoría integral debe comprender la ejecución de un trabajo con los alcances de: auditoría financiera, auditoría de cumplimiento, auditoría de gestión y auditoría de control interno.(Ochoa, 2019). Menciona a la auditoría integral como "evaluación preventiva, permite validar los sistemas administrativos, financiero, de cumplimiento y de gestión que gobiernan una entidad, con el objeto de proponer alternativas para el logro adecuado de sus fines y/o el mejor aprovechamiento de sus recursos". (Jasmín, 2020). Menciona que el propósito de la auditoría integral es evaluar la gestión administrativa y financiera y verificar el cumplimiento de las leyes, reglamentos y normativa aplicables a la entidad.

\section{Teoría de contratación de servidores públicos}


Con relación a las teorías de la contratación de servidores públicos, según Ley del servicio civil Ley $\mathbf{N}^{\circ} \mathbf{3 0 0 5 7}$, Ley que establece un régimen único y exclusivo para las personas que prestan servicios en las entidades públicas del Estado, así como para aquellas personas que están encargadas de su gestión, del ejercicio de sus potestades y de la prestación de servicios a cargo de estas y que las entidad públicas del Estado alcancen mayores niveles de eficacia y eficiencia, y presten efectivamente servicios de calidad a través de un mejor Servicio Civil, así como promover el desarrollo de las personas que lo integran. (Lopez, 2011). Realiza un análisis, interpreta y critica la relación especial de sujeción del servidor público que desarrolla funciones respecto a la contratación estatal en aspectos relevantes y que impliquen una omisión a su deber funcional dentro de la actividad. (Zuleta, 2015). Precisó que la contratación de los servidores públicos, muchas veces se prestan para permear por estrategias sería de gran relevancia endurecer las leyes que la castigan y crear programas educativos donde la ética y la moral sean incluidas de manera transversal durante el proceso de aprendizaje, para que de forma natural cada uno actúe dentro del cumplimiento de los valores humanos, con responsabilidad hacia su propia nación.

Problema General. ¿En qué medida se relaciona la auditoría integral en la contratación de servidores públicos en el Gobierno Regional de Amazonas Periodo 2020?. La justificación teórica es dar a conocer los diferentes conceptos y teorías que refuercen a las entidades que carecen de un plan estratégicos con el propósito de tomar decisiones oportunas y mitigar riesgos. También se justificó de forma práctica porque la presente investigación va a permitir que los servidores públicos tengan conocimiento de la correlación que hay entre la auditoría integral y la contratación de servidores públicos, mitigando todo tipo de riesgos que impidan cumplir con los objetivos del ente. 
Con respecto a la justificación metodológica nos permitirán conocer mejor su accionar y su importancia dentro de las decisiones que se toman en beneficio y mejora dentro de las instituciones del estado para un mejor manejo de los recursos que son asignados; se utilizará correctamente el instrumento de medición la cual es una encuesta que será validada por juicio de expertos. Dadas las razones expuestas conlleva a plantear el objetivo general: Determinar la relación de la Auditoría Integral en la contratación de servidores públicos en el Gobierno Regional de Amazonas Periodo 2020; de igual manera se planteó la hipótesis general: La Auditoría Integral se relaciona con el proceso de contratación de servidores públicos en el Gobierno Regional de Amazonas_2020

\section{MATERIALES Y MÉTODOS}

La presente investigación fue de enfoque cuantitativo, porque la recolección de datos es numérica, estandarizada y cuantificable, y el análisis de la información y la interpretación de resultados permiten fundamentar la comprobación de una hipótesis mediante procedimientos estadísticos, los cuales ofrecen la posibilidad de generalizar los resultados, el tipo de investigación fue básica porque contribuye al conocimiento de la problemática de (Hernández, Fernández y Baptista, 2014). El tipo de investigación fue básica porque contribuye al conocimiento de la problemática de estudio (Hernández, Fernández y Baptista 2010). El diseño de la investigación es no experimental, porque no se van a alterar las variables de estudio, según (Hernández, Fernández y Baptista 2010). "La investigación no experimental, es aquella que se realiza sin manipular deliberadamente variables y en la que sólo se observan los fenómenos en su ambiente natural para después analizarlos", y de corte transversal; ya que se realizó en un momento único. (Hernández et al, 2006). Así mismo es correlacional, por 
estudiar la relación entre variables en una investigación. (Hernández, Fernández, y Baptista, 2014). El método fue hipotético deductivo, ya que se probaron las hipótesis y se obtuvo conclusiones. (Hernández et al, 2006).

Con respecto a la población está comprendida por 60 servidores del gobierno regional de amazonas del 2020, con respecto a los instrumentos utilizados para medir las variables fueron validados por juicio de expertos, se verificaron los contenidos y la estructura de los ítems, también se efectúo la validez de constructo y de criterio,

Para establecer la confiabilidad del instrumento de investigación se hizo uso del coeficiente Alfa de Cronbach, que es un modelo que tiene como propósito medir el índice de consistencia interna, donde sus valores oscilan entre 0 y 1 , y permite estimar la confiabilidad de un instrumento.

Tabla 1.

Resumen de procesamiento de casos

\begin{tabular}{llll}
\hline & & $\mathrm{N}$ & $\%$ \\
\hline Caso & Válido & 20 & 100,0 \\
$\mathrm{~s}$ & Excluido $^{\mathrm{a}}$ & 0 &, 0 \\
& Total & 20 & 100,0 \\
\hline
\end{tabular}

a. La eliminación por lista se basa en todas las variables del procedimiento.

Tabla 2 .

Estadísticas de fiabilidad

Alfa de Cronbach N de elementos

,910 26

El valor en cuantía que más se aproxime a el valor máximo, 1, mayor es la desconfianza. Así pues, en ese sentido se considera que los valores del alfa 
superiores a 0,7, son bastantes para sancionar la fiabilidad. Teniendo así que nuestro instrumento es 0.910 , por lo que concluimos que es excelente

\section{RESULTADOS}

Para los niveles de variables y dimensiones y la estadística inferencia para contratación de hipótesis se aplicó estadística descriptiva, también se realizó el método de baremación para las variables auditoría integral y contratación de servidores públicos, con el fin de determinar los rangos y niveles de los resultados, se tomó

en cuenta la cantidad de preguntas y la escala de likert aplicada en el instrumento

Tabla 3

La baremación de variables

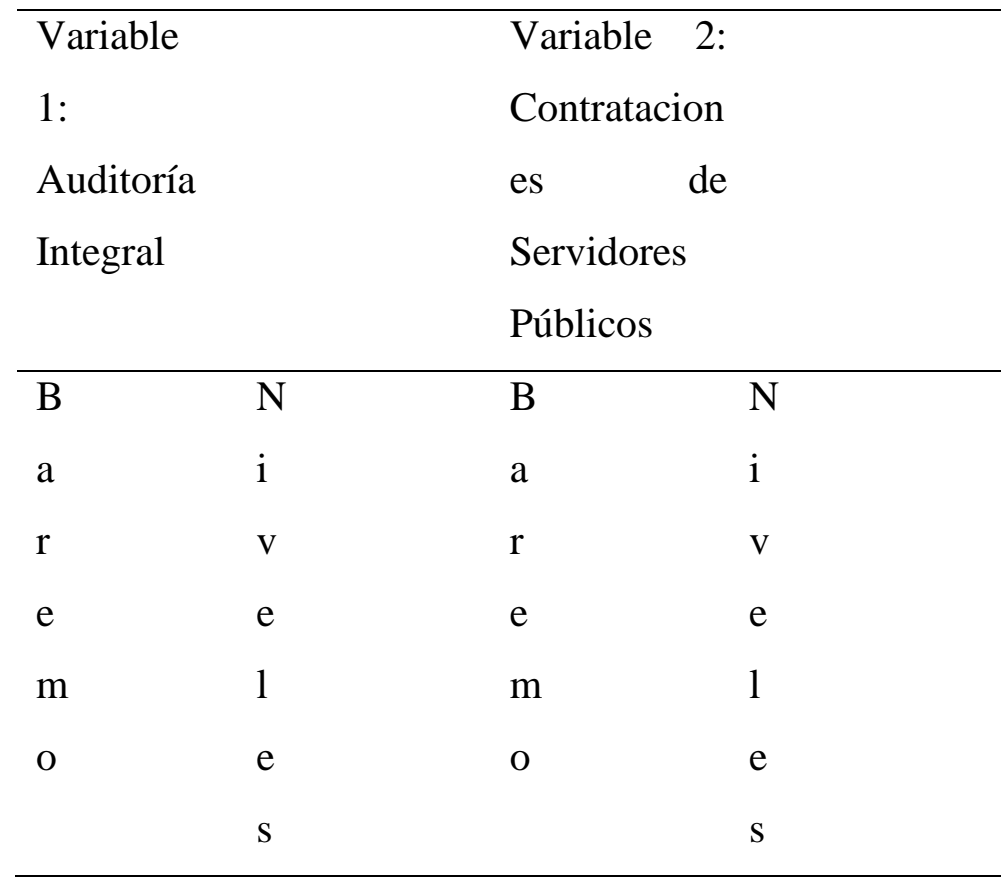


Formación docente

revista iberoamericana de educación

\begin{tabular}{|c|c|c|c|}
\hline 1 & $\mathrm{M}$ & 1 & I \\
\hline 5 & $\mathrm{a}$ & 1 & $\mathrm{n}$ \\
\hline- & 1 & - & $\mathrm{a}$ \\
\hline 3 & o & 2 & $\mathrm{~d}$ \\
\hline 4 & $\mathrm{R}$ & 5 & $\mathrm{e}$ \\
\hline 3 & $\mathrm{e}$ & 2 & $\mathrm{c}$ \\
\hline 5 & $\mathrm{~g}$ & 6 & $\mathrm{u}$ \\
\hline - & $\mathrm{u}$ & - & $\mathrm{a}$ \\
\hline 5 & 1 & 4 & $\mathrm{~d}$ \\
\hline 4 & $\mathrm{a}$ & 0 & o \\
\hline 5 & $\mathrm{r}$ & 4 & A \\
\hline 5 & B & 1 & $\mathrm{~d}$ \\
\hline- & $\mathrm{u}$ & - & e \\
\hline 7 & $\mathrm{e}$ & 5 & $\mathrm{c}$ \\
\hline \multirow[t]{13}{*}{5} & $\mathrm{n}$ & 5 & $\mathrm{u}$ \\
\hline & O & & $\mathrm{a}$ \\
\hline & & & $\mathrm{d}$ \\
\hline & & & o \\
\hline & & & $\mathrm{M}$ \\
\hline & & & $\mathrm{u}$ \\
\hline & & & $\mathrm{y}$ \\
\hline & & & $\mathrm{a}$ \\
\hline & & & $\mathrm{d}$ \\
\hline & & & e \\
\hline & & & $\mathrm{c}$ \\
\hline & & & $\mathrm{u}$ \\
\hline & & & $\mathrm{a}$ \\
\hline
\end{tabular}


d

O

Fuente propia

Luego de realizar la baremación se procedió a analizar los datos recolectados

por medio del software SPSS 24, se obtuvieron los siguientes resultados para

la variable Auditoría Integral:

Tabla 4.

Resultados de la Variable Auditoria Integral

Frecuenci Porcen

\begin{tabular}{lll} 
Niveles & $\mathrm{a}$ & taje \\
\hline $\mathrm{B}$ & 60 & 100,0 \\
$\mathrm{u}$ & & \\
$\mathrm{e}$ & & \\
$\mathrm{n}$ & & \\
$\mathrm{o}$ & &
\end{tabular}

Fuente: Encuesta aplicada

Como se aprecia en la tabla4, el 100,0 \% de los servidores del Gobierno

Regional de Amazonas percibe que la Auditoría Integral es bueno.

Así también se obtuvieron los siguientes resultados para la variable contratación de servidores públicos:

Tabla 5.

Resultados de la variable Contrataciones de Servidores Públicos

Porcenta

Niveles Frecuencia je 
$\mathrm{Mu}$

60

100,0

$\mathrm{y}$

ade

cua

do

Fuente: Encuesta aplicada

Como se aprecia en la tabla 5, el 100,0 \% de los servidores del Gobierno

Regional de Amazonas percibe que la contratación de servidores públicos es muy adecuado.

En parte de la estadística inferencial, determinamos la prueba de bondad de ajuste de nuestra variables, Auditoría Integral y Contratación de servidores públicos

Tabla 6.

Pruebas de normalidad de las variables y dimensiones

\begin{tabular}{|c|c|c|}
\hline \multicolumn{3}{|c|}{ Kolmogorov-Smirnov $^{\mathrm{a}}$} \\
\hline \multicolumn{3}{|l|}{$\mathrm{E}$} \\
\hline \multicolumn{3}{|l|}{ st } \\
\hline \multicolumn{3}{|l|}{$\mathrm{a}$} \\
\hline \multicolumn{3}{|l|}{$\mathrm{d}$} \\
\hline ís & & $\mathrm{S}$ \\
\hline $\mathrm{ti}$ & & $\mathrm{i}$ \\
\hline c & $\mathrm{g}$ & $\mathrm{g}$ \\
\hline o & 1 & . \\
\hline
\end{tabular}


Auditoría integral

Auditoría financiera

Auditoría de gestión

Auditoría
cumplimiento

de

,

2

7

9

Auditoría de control interno

2

0

8

Contratación

servidores públicos

de

Preparatoria

$\begin{array}{ll}6 & \\ 0 & 1 \\ & 9 \\ & 5\end{array}$

6

0

0

0

0

6

0

0

0

0

6

0

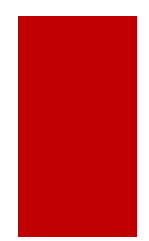

6

0

0

0

0

6

0

0

0

0

6

0

0

0

0 
Convocatoria

Selección
6

0

0

3

1

$\begin{array}{lll}, & 6 & \\ 1 & 0 & 0 \\ 9 & & 0 \\ 6 & & 0\end{array}$

*. Esto es un límite inferior de la significación verdadera.

a. Corrección de significación de Lilliefors

Fuente: Software estadístico

Como se aprecia de la tabla 6, la prueba de normalidad Kolmogórov Smirnov.

El valor de probabilidad para la variable 01 Auditoria Integral $p>0,05$ por lo tanto tiene una distribución normal, asimismo para la variable 02 Contratación de Servidores Públicos; apreciamos que el valor de p<0,05. Concluimos que la distribución muestral no es normal.

En referencia a la estimación de la relación entre variable: Considerando que las distribuciones muestrales no siguen una distribución normal para hallar la correlación entre variables usamos la estadística de correlación de Spearman

$$
r_{s}=1-\frac{6 \sum_{i=1}^{n} d_{i}^{2}}{n\left(n^{2}-1\right)} \quad \text { además } \quad-1 \leq r_{s} \leq 1
$$

Así pues, luego de aplicar la formula sobre las puntuaciones de ambas variables se obtiene el siguiente resultado:

rho $=-0,041=4,1 \%(* *)$ además $-1 \leq \mathrm{rs} \leq 1$

tal como lo muestra en la figura $\mathrm{N}^{\circ} 1$

Figura 1.

Diagrama de dispersión de la correlación de las variables en estudio 


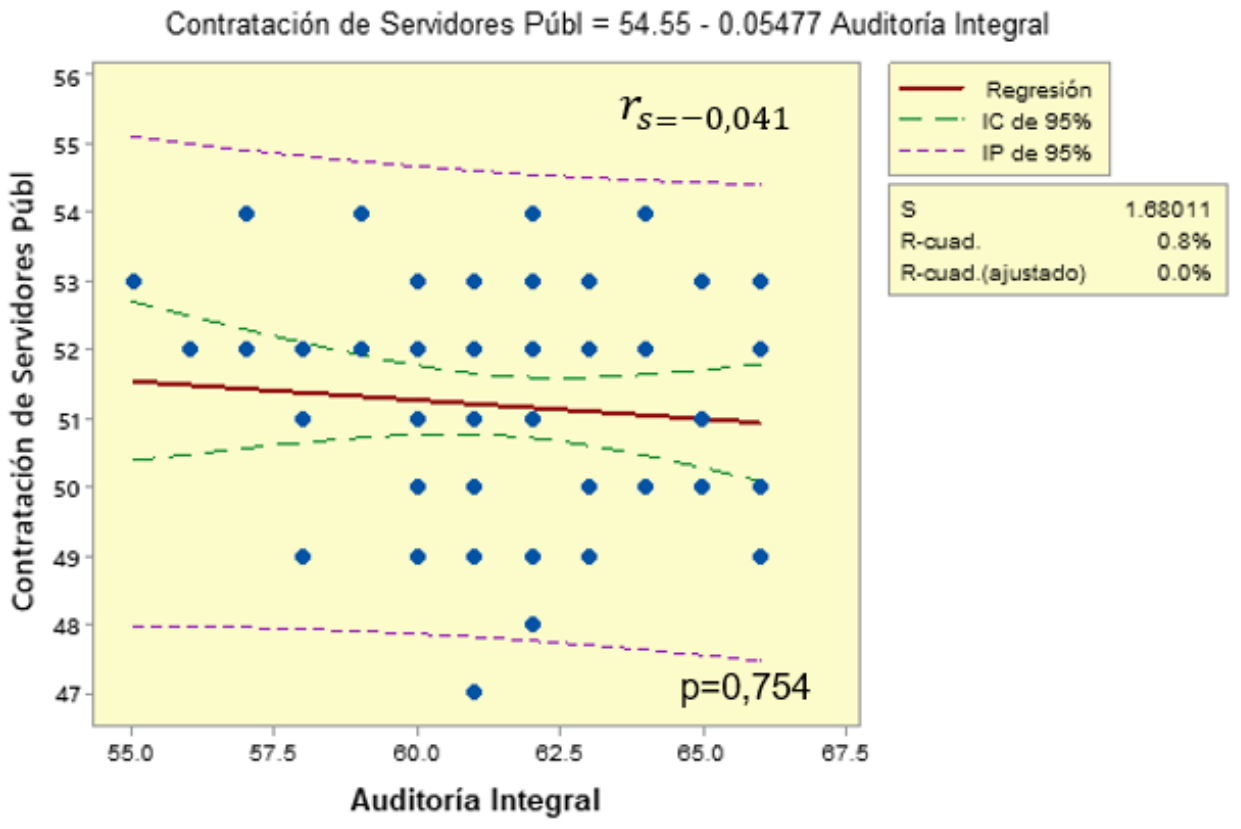

Fuente: software estadístico

Su relación categórica de las variables, se presenta en la tabla 7:

Tabla 7.

Resultados de la relación categórica para las variables Auditoría Integral y

la Contratación de Servidores Públicos

Auditoría Integral

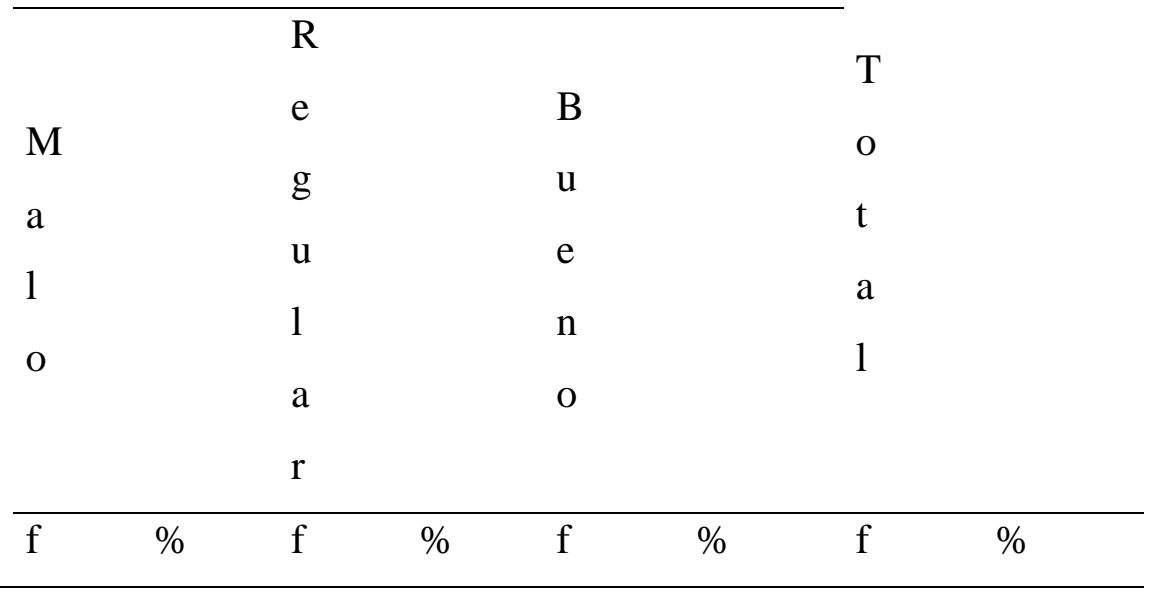




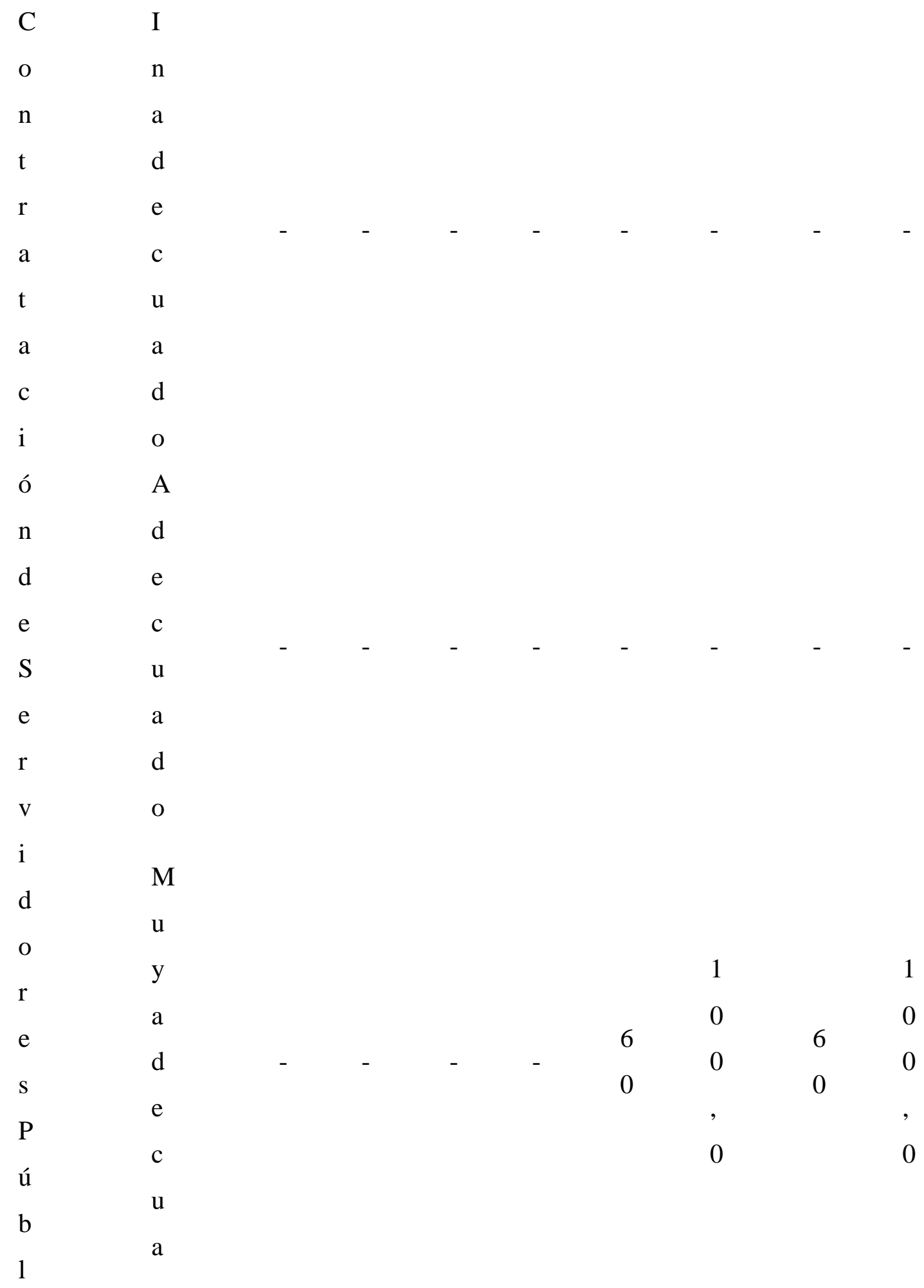




$\begin{array}{ll}\text { i } & \text { d } \\ \text { c } & \text { o } \\ \text { o } & \\ \text { s } & \end{array}$

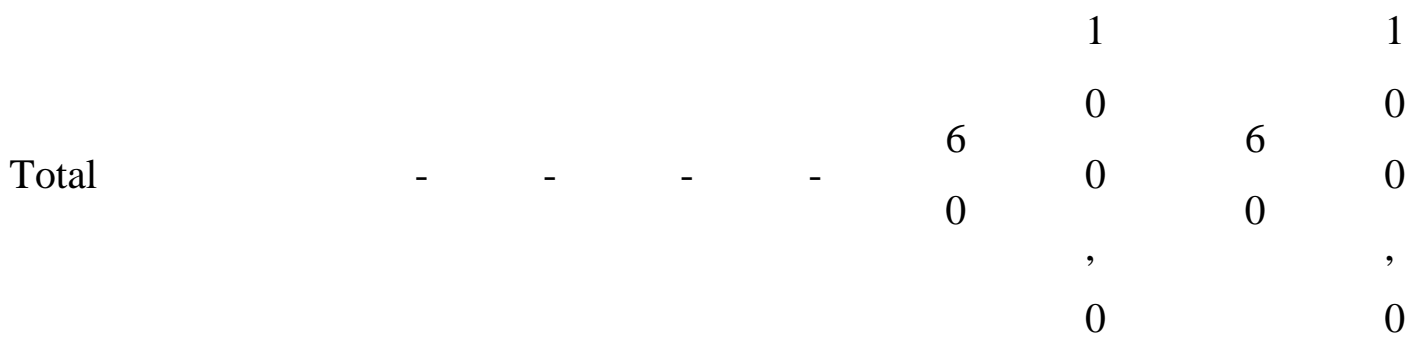

\author{
d
}

O

Fuente: Instrumento aplicado

En la tabla 7 apreciamos la tabla de contingencia de las variables en estudio la Auditoría Integral y la Contratación de Servidores Públicos, entonces del total de encuestados; el 100,0\% (60) de casos perciben que la Auditoría Integral es bueno y la Contratación de Servidores Públicos es muy adecuado.

\title{
Proceso de la prueba de Hipótesis
}

Ho. La Auditoría Integral no se relaciona de forma directa con el proceso de contratación de servidores públicos en el Gobierno Regional de Amazonas 2020. $\rho_{s}=0$

Hi. La Auditoría Integral se relaciona de forma directa con el proceso de contratación de servidores públicos en el Gobierno Regional de Amazonas 2020. $\rho_{s}>0$

Siendo $\rho_{s}$ : Correlación poblacional por rangos.

Estadística de Prueba. $z=r_{s} \sqrt{n-1}$

Cálculo de la estadísitica. $z=-0,041 \sqrt{60-1}=-0,315$ 


\section{Toma de desiciones}

El valor calculado lo tabulamos en la figura, de la cual podemos deducir que el valor calculado se ubica en la región crítica $(-0,315>-1,645)$ por lo que diremos que se ha encontrado evidencia empírica para rechazar la hipótesis alterna y aceptar la hipótesis nula, es decir:

La Auditoría Integral no se relaciona de forma directa con el proceso de contratación de servidores públicos en el Gobierno Regional de Amazonas 2020

Figura2.

Diagrama de densidad de la distribución normal

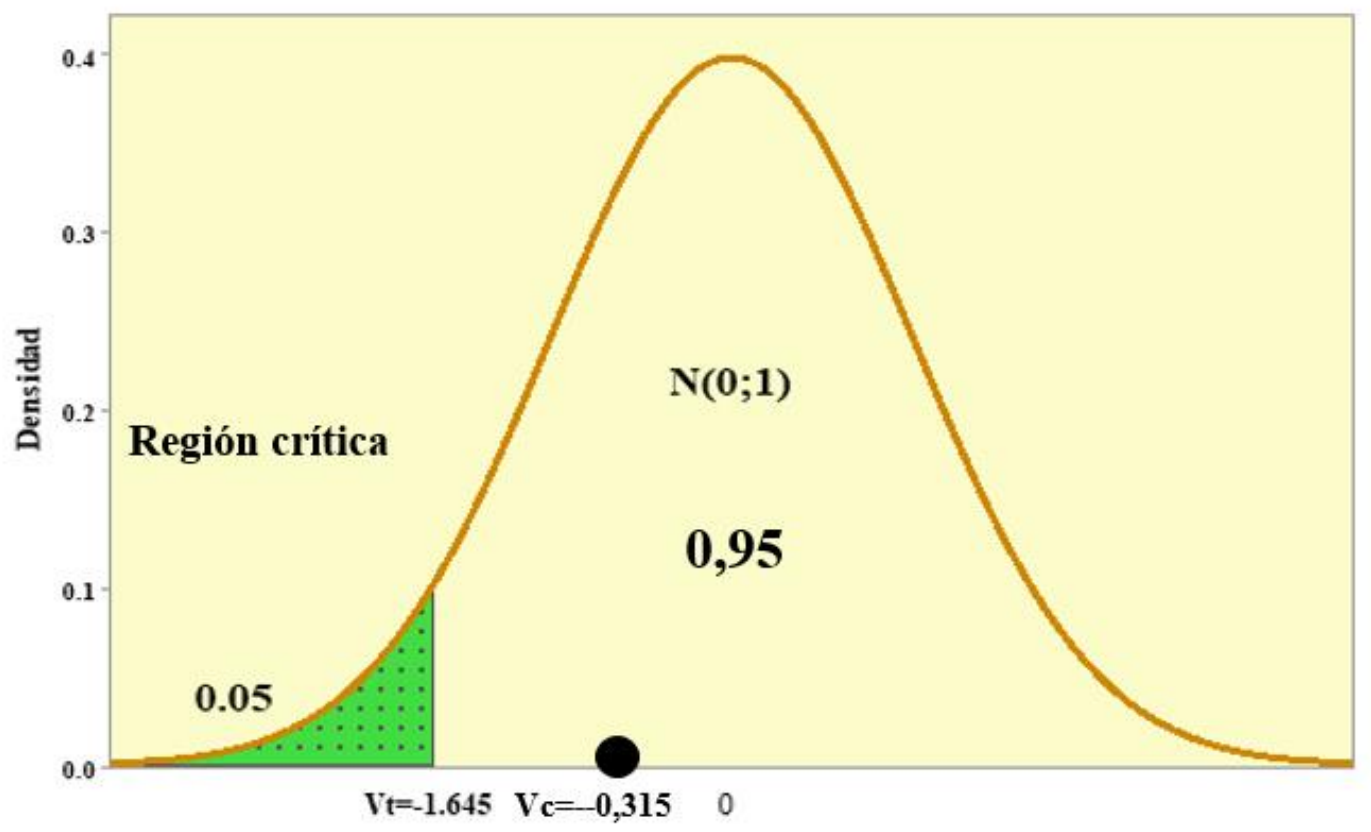

Prueba de la significancia de las hipótesis especificas

Tabla 8.

Estadísticas para la prueba de las hipótesis específicas 


\begin{tabular}{|c|c|c|c|c|c|}
\hline \multirow{2}{*}{$\begin{array}{l}\text { Dimensiones } \\
\text { De la Auditoría } \\
\text { Integral }\end{array}$} & \multirow[t]{2}{*}{ ticas de la } & \multirow[b]{2}{*}{$V_{C}=z$} & \multirow[t]{2}{*}{ Contratac } & \multirow[b]{2}{*}{$V_{C}>V_{z . c r i}$} & \multirow[t]{2}{*}{ dores públicos } \\
\hline & & & & & \\
\hline Auditoría financiera & $-0,250$ & $-1,920$ & $-1,645$ & True & Rechazo Ho \\
\hline Auditoría de gestión & $-0,082$ & $-0,630$ & $-1,645$ & False & Rechazo H1 \\
\hline Auditoría de cumplimiento & $-0,102$ & $-0,783$ & $-1,645$ & False & Rechazo H1 \\
\hline Auditoría de control interno & 0,221 & 1,700 & $-1,645$ & True & Rechazo Ho \\
\hline
\end{tabular}

Software estadístico.

De la Tabla 8 observamos que el valor calculado de la correlación es rs $=-0$, 250 con $\mathrm{p}=0,054(\mathrm{p}>0,05)$ la misma que tiene asociado un valor calculado de $\mathrm{z}=-1,920$ y el valor critico de $-1,645$ (obtenido de las tablas estadísticas) de tal manera que se cumple la relación $(-1,920>-1,645)$ por lo tanto procedemos a rechazar la hipótesis nula y aceptar la hipótesis alterna con un 95\% de confianza

De la Tabla 8 observamos que el valor calculado de la correlación es rs $=-0$, 082 con $\mathrm{p}=0,534$ ( $\mathrm{p}>0,05)$ la misma que tiene asociado un valor calculado de $\mathrm{z}=-0,630$ y el valor critico de $-1,645$ (obtenido de las tablas estadísticas) de tal manera que se cumple la relación $(-0,630<-1,645)$ por lo tanto procedemos a rechazar la hipótesis alterna y aceptar la hipótesis nula con un 95\% de confianza

De la Tabla 8 observamos que el valor calculado de la correlación es rs= $0,102$ con $\mathrm{p}=0,438$ ( $\mathrm{p}>0,05)$ la misma que tiene asociado un valor calculado de $\mathrm{z}=-0,438$ y el valor critico de $-1,645$ (obtenido de las tablas estadísticas) de tal manera que se cumple la relación $(-0,438<-1,645)$ por lo tanto procedemos a rechazar la hipótesis alterna y aceptar la hipótesis nula con un $95 \%$ de confianza. 
De la Tabla 8 observamos que el valor calculado de la correlación es rs $=0$, 221 con $\mathrm{p}=0,089(\mathrm{p}>0,05)$ la misma que tiene asociado un valor calculado de $\mathrm{z}=1,700 \mathrm{y}$ el valor critico de $-1,645$ (obtenido de las tablas estadísticas) de tal manera que se cumple la relación $(1,700>-1,645)$ por lo tanto procedemos a rechazar la hipótesis nula y aceptar la hipótesis alterna con un 95\% de confianza

\section{DISCUCIÓN}

De los resultados se evidencio de acuerdo al objetivo general planteado que fue determinar la relación de la Auditoría Integral en la contratación de servidores públicos en el Gobierno Regional de Amazonas Periodo 2020; mediante el cálculo de la estadística correlación de "rho" de Spearman cuyo valor obtenido es de rs=- 0,041 con ( $\mathrm{p}=0,754)$; que es la evidencia para el cumplimiento del objetivo general de la investigación. Asimismo, el diagrama de dispersión nos muestra que los puntos se agrupan alrededor de la línea oblicua lo cual nos confirma la presencia de una relación inversa entre las variables Auditoría Integral en la Contratación de Servidores Públicos.

Asimismo, los resultados muestran que para la variable 01 Auditoria Integral el nivel que tiene más relevancia es el nivel bueno con un 100,0\% del total de encuestados. Respecto a sus 4 dimensiones. Para la auditoría financiera la que tiene más relevancia es el nivel bueno con un 100,0\%. Para la auditoría de gestión prevalece el nivel bueno con un $81.7 \%$. Para la auditoría de cumplimiento prevalece el nivel regular con un $90,0 \%$ y finalmente para la auditoría de control interno prevalece el nivel bueno con un 100,0\% del total de los servidores públicos del Gobierno Regional de Amazonas. 
En tanto para la variable 02 Contratación de Servidores Públicos, los resultados muestran que los servidores del Gobierno Regional de Amazonas, es muy adecuado en un $100,0 \%$ de casos.

Para el cumplimiento del primer objetivo específico, de la Tabla 13 muestra los respectivos resultados. La correlación obtenida entre la dimensión auditoría financiera se relaciona de forma directa con la contratación de servidores públicos cuyo resultado es de $\mathrm{rho}=-0,250$ con $\mathrm{p}=0,054(\mathrm{p}>0,05)$ es la evidencia para el cumplimiento del primer objetivo de la investigación. Para el cumplimiento del segundo objetivo específico, de la Tabla 13 muestra los respectivos resultados. La correlación obtenida entre la dimensión auditoría de gestión se relaciona de forma directa con la contratación de servidores públicos cuyo resultado es de rho $=-0,082$ con $p=0,534(p>0,05)$ que es la evidencia para el cumplimiento del segundo objetivo de la investigación.

Para el cumplimiento del tercer objetivo específico, de la Tabla 08 muestra los respectivos resultados. La correlación obtenida entre la dimensión auditoria de cumplimiento se relaciona de forma directa con la contratación de servidores públicos cuyo resultado es de rho $=-0,102$ con $p=0,438(p>0,05)$ que es la evidencia para el cumplimiento del tercer objetivo de la investigación.

Finalmente, para el cumplimiento del cuarto objetivo específico, de la Tabla 08 muestra los respectivos resultados. La correlación obtenida entre la dimensión auditoria del control interno se relaciona de forma directa con la contratación del control interno cuyo resultado es de rho $=0,221$ con $\mathrm{p}=0,089$ $(p>0,05)$ que es la evidencia para el cumplimiento del quinto objetivo de la investigación. 
Asimismo, en referencia a las variables estudiadas al contrastarlos y se han examinado y tomado en cuenta las siguientes investigaciones previas del ámbito internacionales relacionados a la auditoría integral, y de acuerdo con Acosta (2011), “en su investigación menciona los métodos bibliométricos y técnicas de visualización basadas en el análisis de redes sociales para analizar y procesar los datos; el análisis-síntesis para conformar la base teórica y metodológica, así como el sistémico-estructural para abordar las relaciones entre auditorías de información y auditorías de conocimiento, con un enfoque integral”. En tanto Cedeño( 2016), desde el punto de vista metodológico “ esta investigación genera la aplicación de conocimiento válido y confiable para TSC en sus áreas administrativas financiera, mediante la adopción de un instrumento como lo auditoría integral para evaluar su desempeño. La misma se realizará considerando el proceso de investigación científica, identificando la problemática y formulando las soluciones correspondientes a través de los hallazgos detectados"; concluye que: la implementación de una Auditoría Integral en TUNASERV Servicios Portuarios S.A., logrará mitigar deficiencias en las áreas contable, comercial y administrativa que afectan de forma negativa el adecuado y eficiente desempeño de la empresa, por lo tanto las capacitación y competencias profesionales son factores fundamentales para el logro de los objetivos institucional, y aplicar los resultados de este trabajo como una herramienta que contribuya en la gestión y toma de decisiones. Asimismo (Veloz, et al 2017), la presente investigación en su metodología es de corte exploratorio, investigación descriptiva, se caracteriza desde la visión sistémica, holística, los componentes del campo de estudio de la auditoría integral, a partir del conocimiento que se adquiere durante la investigación, en una de sus conclusiones considera que la implementación de una Auditoría Integral demuestra validez y pertinencia en calidad de 
herramienta gerencial aplicada a las pequeñas Cooperativas de Ahorro y Crédito, al realizar un diagnóstico de la situación actual, se evalúa y se plantea un plan de mejoras para llegar al cumplimiento de los objetivos de la organización. (Bautista, 2015), concluye que es importante establecer la enseñanza ética en la formación de los servidores públicos en tres momentos fundamentales: Durante la formación académica; mediante el proceso de Inducción al servicio público y mediante un sistema basado en calidad de Capacitación y Desarrollo de Personal. Asimismo, Vaca (2017). Menciona como objetivo de relacionar los factores de motivación laboral que describen el comportamiento de los servidores públicos en la última década, a través del análisis de varias teorías de motivación que permitan determinar los factores que más motivan a un servidor público del país. A partir de los resultados se discuten las consecuencias y la construcción de diseños de modelos teóricos que apuntan finalmente a líneas de investigación futuras. En referencia, a los trabajos previos nacionales podemos mencionar: Guardia (2020), Quien estableció la relación entre variables; su población fue conformada por 135 participantes y llegó a la siguiente conclusión: La manera como alcanzar un alto grado de suficiencia en la auditoría integral de las empresas; es mediante la mayor cantidad de papeles de trabajo, porque de esa forma se obtiene la información que deberá compararse con los criterios correspondientes. De la misma manera, Campos (2019), determinó un método descriptivo y explicativo, siendo su población de 42 trabajadores entre, directivos, funcionarios y trabajadores del área de abastecimientos y servicios auxiliares de la Universidad Nacional Daniel Alcides Carrión. Asimismo, Aspajo (2012), en el presente trabajo de investigación corresponde al diseño descriptivo explicativo causal compuesta, no experimental, por ser eminentemente de causa - efecto entre la auditoría integral y la dirección financiera del Hospital Carlos Monge Medrano de la ciudad de Juliaca en el 
año 2016, su población fue de 69 trabajadores. Príncipe (2019), Fue una investigación descriptiva inferencial. La muestra fue de 120 personas y se identificó que hay una relación regular

\section{CONCLUSIONES}

Se ha determinado la relación de la Auditoría Integral en la contratación de servidores públicos en el Gobierno Regional de Amazonas Periodo 2020. El cálculo de la estadística correlación de "rho" de Spearman cuyo valor obtenido es de rho $=-0,041$ con $p=0,754(p>0,05)$, que es la evidencia para el cumplimiento del objetivo general de la investigación.

Se ha determinado la relación de la Auditoría Financiera en la contratación de servidores públicos en el Gobierno Regional de Amazonas Periodo 2020.El cálculo de la estadística correlación de "rho" de Spearman cuyo valor obtenido es de rho $=-0,250$ con $p=0,054(p>0,05)$, que es la evidencia para el cumplimiento del primer objetivo especifico.

Se ha determinado la relación de la Auditoría de Gestión en la contratación de servidores públicos en el Gobierno Regional de Amazonas Periodo 2020.El cálculo de la estadística correlación de "rho" de Spearman cuyo valor obtenido es de rho $=-0,082$ con $\mathrm{p}=0,534(\mathrm{p}>0,05)$, que es la evidencia para el cumplimiento del segundo especifico.

Se ha determinado la relación de la Auditoría de Cumplimiento en la contratación de servidores públicos en el Gobierno Regional de Amazonas Periodo 2020. El cálculo de la estadística correlación de "rho" de Spearman cuyo valor obtenido es de $\mathrm{rho}=-0,102$ con $\mathrm{p}=0,438(\mathrm{p}>0,05)$, que es la evidencia para el cumplimiento del tercer objetivo especifico.

Se ha determinado la relación de la Auditoría de Control Interno en la contratación de servidores públicos en el Gobierno Regional de Amazonas Periodo 2020. El cálculo de la estadística correlación de "rho" de Spearman 
cuyo valor obtenido es de rho $=0,221$ con $\mathrm{p}=0,089(\mathrm{p}>0,05)$, que es la evidencia para el cumplimiento del cuarto objetivo especifico.

Se ha determinado la relación del subsistema estructural administrativo con la ejecución del proceso del Presupuesto Participativo en el Gobierno Regional de Huancavelica, año 2020. El cálculo de la estadística correlación de "rho" de Spearman cuyo valor obtenido es de rho $=0,452 * *$ con $\mathrm{p}=0,000(\mathrm{p}<0,05)$ tipificada como positiva débil, que es la evidencia para el cumplimientonto del cuarto objetivo especifico.

\section{REFERENCIAS}

Acosta-Palmer, H. R., \& Troncoso-Fleitas, M. D. L. C. (2011). Auditoria integral de mantenimiento en instalaciones hospitalarias, un análisis objetivo. Ingeniería Mecánica, 14(2), 107-118. https://scholar.google.com.pe/scholar?hl=es\&as_sdt=0\%2C5\&as_vis=1\&q= auditoria+integral+tesis+internacional $\& b \operatorname{tnG}=\& o q=$ auditoria+integ

Álvarez, Marivel Huamaní. «TESIS PARA OPTAR EL TÍTULO PROFESIONAL DE CONTADOR PÚBLICO», 2015, 98.

«aspajo_tn.pdf». Accedido 28 de diciembre de 2020. http://repositorio.usmp.edu.pe/bitstream/handle/20.500.12727/1126/aspajo_t n.pdf?sequence $=1 \&$ isAllowed=y.

«Auditoria Integral LIBRO.pdf», s. f.

«Auditoria Integral.pdf». Accedido 28 de diciembre de 2020. https://digitk.areandina.edu.co/bitstream/handle/areandina/1268/Auditoria\% 20Integral.pdf? sequence $=1 \&$ is Allowed $=\mathrm{y}$. 
Bautista, E. (2009). La auditoría integral. El Cid Editor. https://scholar.google.es/scholar?hl=es\&as_sdt=0\%2C5\&q=articulo+cientifi co\%2C+auditor\%C3\%ADa+integral\&btnG=

Cantos Ochoa, Marcos Eduardo. «La auditoría integral como herramienta de validación de la gestión institucional». Telos 21, n. ${ }^{\circ} 2$ (8 de mayo de 2019): 422-48. https://doi.org/10.36390/telos212.09.

Cárdenas, Navarro, Juan Carlos, Osejo Domínguez, y Edgar Efraín. «TRABAJO DE FIN DE MAESTRÍA», s. f., 147.

Cedeño-Choéz, P. J. (2016). Auditoría integral a los activos fijos depreciables. Dominio de las Ciencias, 2(2), 406-417. https://scholar.google.es/scholar?hl=es\&as_sdt=0\%2C5\&q=articulo+cientifi co $\% 2 \mathrm{C}+$ auditor $\% \mathrm{C} 3 \% \mathrm{ADa}+$ integral\&btnG=

De La Torre Lascano, Carlos Mauricio. «Interrelación actual entre Gobierno Corporativo, Respondabilidad y Auditoría Integral: una visión reformulada». Telos Revista de Estudios Interdisciplinarios en Ciencias Sociales 20, n. 3 (14 de septiembre de 2018): 492-509. https://doi.org/10.36390/telos203.06.

«LEGIS Xperta|Plataforma digital con soluciones profesionales». Accedido 28 de diciembre de 2020. https://xperta.legis.co/visor/temp_rcontador_04eed461-9ff8-4622-b6d635d5da610f5a.

Luna, Yanel Blanco. Auditoría integral: normas y procedimientos. Ecoe Ediciones, 2015. 
Mejías, Néstor D, y Bruno Rezzoagli. «Auditoría de Gestión Integral. Nuevos aportes para la evaluación y el control de la Planificación Institucional. El caso de la Universidad Nacional del Litoral», 2017, 4.

Polar Falcón, Ernesto Augusto. «UN NUEVO ENFOQUE DE LA AUDITORIA INTEGRAL PARA SU APLICACIÓN EN EL PERÚ». Quipukamayoc 7, n. ${ }^{\circ} 14 \quad(19$ de marzo de 2014): 41. https://doi.org/10.15381/quipu.v7i14.5696.

Rozas Flores, Alan Errol. «AUDITORÍA iNT6EGRAL. Nueva Visión de su alcance». Quipukamayoc 5, n. ${ }^{\circ} 10$ (22 de marzo de 2014): 111. https://doi.org/10.15381/quipu.v5i10.6006.

«TESIS POSGRADO ABRAHAM BONILLA -SUNEDU.pdf». Accedido 10 de diciembre de 2020. http://repositorio.undac.edu.pe/bitstream/undac/899/1/TESIS\%20POSGRA DO\%20ABRAHAM\%20BONILLA\%20-SUNEDU.pdf.

«TESIS YACHACHIN PANEZ.pdf». Accedido 10 de diciembre de 2020. http://repositorio.undac.edu.pe/bitstream/undac/558/1/TESIS\%20YACHAC HIN\%20PANEZ.pdf

Luna, Y. B. (2015). Auditoría integral: normas y procedimientos. Ecoe ediciones. https://n9.cl/02zf8

Ochoa, M. E. C. (2019). La auditoría integral como herramienta de validación de la gestión institucional. Telos: Revista de Estudios Interdisciplinarios en Ciencias Sociales, 21(2), 422-448. https://n9.cl/hyj4s 
Hernández, R., Fernández, C. Y Baptista, L. (2014). Metodología de la Investigación. 6a . Edición, Editorial Mc Grawn Hill, México.

http://dx.doi.org/10.19053/20278306.4602

Jasmin, M., \& Cisneros, S. (2020). “Auditoría integral y su incidencia en la gestión administrativa y financiera de la ctic, cantón Caluma, período 2018”' (Bachelor's thesis, Universidad Nacional de Chimborazo).

López Pedraza, H. A., \& Yara Delgado, S. J. (2011). Responsabilidad de los Servidores Públicos en el Desarrollo de la Contratación Estatal.

Vaca, M. (2017). Motivación Laboral en los servidores públicos de Ecuador. INNOVA Research Journal, 2(7), 101-108. https://n9.cl/wtgks

Zuleta, A. P. (2015). La corrupción su historia y sus consecuencias en Colombia. https://n9.cl/kz15

Fernando, V. N., Diana, V. U., \& Cesar, V. M. (2017). Modelo de auditoría integral para pequeñas Cooperativas de Ahorro y Crédito ecuatorianas. Revista Ciencia UNEMI, 10(23), 49-56. https://scholar.google.es/scholar?hl=es\&as_sdt=0\%2C5\&q=articulo+cientifi co\%2C+auditor\%C3\%ADa+integral\&btnG= 